\title{
Student interpretations of uncertainty in classical and quantum mechanics experiments
}

\author{
Martin M. Stein, ${ }^{1}$ Courtney White, ${ }^{2}$ Gina Passante, ${ }^{2}$ and N. G. Holmes ${ }^{1}$ \\ ${ }^{1}$ Laboratory of Atomic and Solid State Physics, Cornell University, 245 East Avenue, Ithaca, NY, 14853 \\ ${ }^{2}$ Department of Physics, California State University Fullerton, 800 N. State College Blvd., Fullerton, CA, 92831
}

Measurements in quantum mechanics are often taught in an abstract, theoretical context. Compared to what is known about student understanding of experimental data in classical mechanics, it is unclear how students think about measurement and uncertainty in the context of experimental data from quantum mechanical systems. In this paper, we tested how students interpret the variability in data from hypothetical experiments in classical and quantum mechanics. We conducted semi-structured interviews with 20 students who had taken quantum mechanics courses and analyzed to which sources they attribute variability in the data. We found that in the quantum mechanics context, most students interpret any variability in the data as irreducible and inherent to the theory. While acknowledging the influence of experimenter error, limited resolution of measurement equipment, and confounding variables (like air resistance) in classical mechanics, many students did not recognize the influence of such effects in quantum mechanics. Some students expressed the view that there are inherently fewer confounding variables in Quantum Mechanics and the equipment used is more precise. We derive tentative implications for instruction and propose further research to test the influence of framing on the responses to our interview protocol.

2019 PERC Proceedings edited by Cao, Wolf, and Bennett; Peer-reviewed, doi.org/10.1119/perc.2019.pr.Stein Published by the American Association of Physics Teachers under a Creative Commons Attribution 4.0 license. Further distribution must maintain attribution to the article's authors, cover page, and DOI. 


\section{INTRODUCTION}

Student understanding of the nature of science and scientific measurement are critical for any science curriculum. In physics, students experience the ideas of measurement in two very different contexts: classical measurement and quantum mechanical measurement. The ways in which measurement and uncertainty are discussed differ significantly between these two contexts. This paper presents preliminary work evaluating the ways students think about measurement in each context. We argue that students use two distinctive ways of reasoning about uncertainty in the context of classical mechanics and quantum mechanics experiments.

In classical mechanics, students typically attribute measurement uncertainty to limitations of the measurement system, conflating statistical variability, systematic effects, and outright mistakes [1]. Research on student understanding of uncertainty has found that students often describe uncertainty according to a point paradigm, where importance is placed on individual measurements, such that any single measurement could be the 'true' value [2]. Often, point reasoning includes beliefs that deviation from a 'true' value is caused by error, interpreted as being a mistake. Set reasoning, in contrast, places importance only on the combined set of measurements, such that the measurements are estimates of the quantity being studied and deviation between measurements is random according to a probability distribution characterizing the measurement process. At the introductory level, many students reason about measurement from both paradigms [24]. In either case, the sources or nature of the uncertainty are more often attributed to the measurement process and rarely attributed to fundamental principle or concepts.

In quantum mechanics, notions of measurement and uncertainty emerge in very different forms. In typical quantum mechanics courses, the term measurement refers to obtaining the eigenvalues of an operator associated with the observable. In most cases, the result of the measurement is not deterministic; rather there is a non-zero probability that several different eigenvalues could result from the measurement. This probabilistic nature of quantum mechanics results in an uncertainty that is inherent to the theory. Additionally, there are relationships that limit the minimum uncertainty between related variables. The position-momentum uncertainty principle is perhaps one of the most well-known equations in all of quantum mechanics.

There has been limited research into student thinking about measurement and uncertainty in quantum mechanics. Most research into measurements has dealt with the calculation of uncertainty from an abstract, theoretical perspective [5-8]). One study found that students describe uncertainty in quantum mechanics in four possible ways: 1) that the uncertainty is due to external variables (such as noise or vibrations or poor equipment); 2) that the uncertainty characterizes departure from the exact value due to measurement limitations; 3 ) that the uncertainty arises when measurement of one variable disturbs the other variable; 4) that the uncertainty is inher- ent to the physical systems [9]. Most students described the uncertainty in the uncertainty principle using the first two descriptions. The fourth description (described as most correct by experts) was selected by the fewest students in the sample and, when selected, usually involved rote descriptions that exhibited little conceptual understanding. Other research has found similar categories of reasoning as well as students characterizing the uncertainty as the standard deviation of repeated trials [10], or due to the fact that the particles are traveling very fast $[6,11]$.

This reasoning about uncertainty in quantum mechanics has several parallels to reasoning about uncertainty in classical mechanics. A direct comparison, however, has not previously been made. Furthermore, the ways in which students think about measurement in quantum mechanics when faced with experimental data as well as the theoretical, quantum mechanical phenomenon is unclear. In this study, we aimed to evaluate the ways in which students think about measurement and uncertainty in classical and quantum mechanical situations when reasoning about hypothetical experimental data. This work aims to contribute to the broader research question: What is the interplay between how students reason about measurement in classical and quantum mechanical contexts? Throughout this work, we aim to evaluate whether instruction in either context can affect student thinking about measurement and uncertainty broadly.

\section{METHODS}

\section{A. Recruitment and Participants}

We recruited participants from courses in quantum mechanics and advanced lab courses to get a population that is familiar with the physical effects that govern the experiments we would cover during the interview. The participants included 18 undergraduate and 2 Masters students from an R1 and R3 institution with very different demographics. One institution is a private, $\mathrm{PhD}$-granting institution with approximately 120 physics majors, the other is a bachelor and masters granting four-year institution, designated as a HispanicServing Institution, with around 100 physics majors.

All students were selected such that they were either currently taking a quantum mechanics class or had taken one in the past. For the purpose of this study, we purposefully chose to recruit students from a range of classes to cover the broad spectrum of perceptions that students have about measurement uncertainty in quantum mechanics.

\section{B. Data Collection and Analysis}

We used structured interviews that presented students with sets of data from two different hypothetical experiments, one classical mechanics and one quantum mechanics experiment. In the classical mechanics context, students were first only 


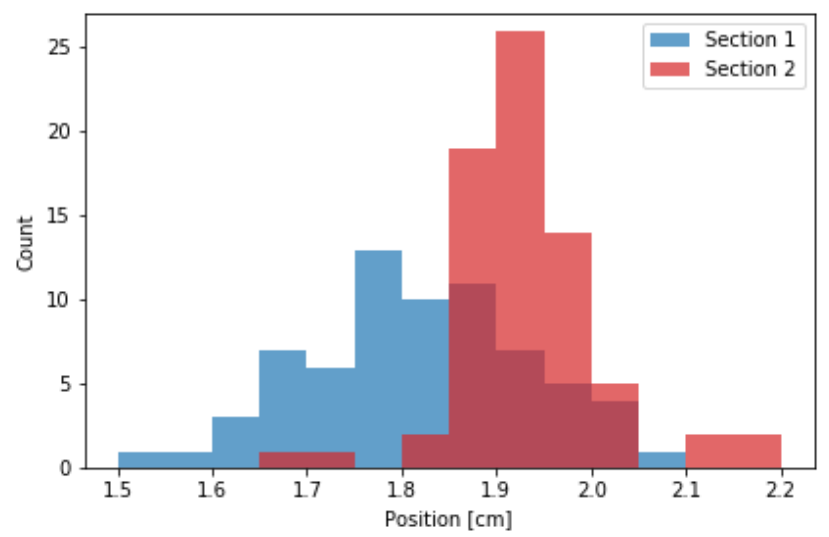

FIG. 1. Data that was provided to students during the interviews in each context. First, we showed students only the blue histogram stemming from lab section 1 and asked questions about the variability. Then we presented them data from lab section 2 and repeated some of the questions. This process was repeated in both contexts, classical and quantum mechanics.

shown the blue histogram from Figure 1. They were given the description: "Suppose you are in a lab investigating the position at which a falling object lands that is released from a ramp. The TA sets up the ramp at the front of the room and asks every student to release a ball several times from the ramp and measure the point where it lands. The students combine their 63 measurements of the position and plot the above histogram." Additionally, the interviewer roughly sketched the experimental setup and specified which quantities were measured. This scenario was adapted from the Physical Measurement Questionnaire [2]. We purposefully did not specify many details of the experimental procedure to see what students would assume and give them the possibility to explore different scenarios (e.g. if participants did not explicitly ask, they were not told if a meter stick or electronic measurement was used to measure where the ball lands). In the quantum mechanics context, the ball-drop experiment was replaced by a single-photon single-slit experiment, but the histogram presented was the same except for a change of units along the horizontal position axis. In both contexts, we asked students the same questions about the uncertainty in the position measurements of the ball or the single photons in the single slit experiment.

All interviews were conducted by the first author. After every interview, the interviewer took notes following a predefined protocol based on [12] to summarize the key ideas the participant expressed. All interviews were transcribed and a subset of questions presented in the Results section were analyzed using the codes in Fig 2. After conducting the interviews and before analyzing them, we decided on codes that classify how students describe uncertainty inspired by the Modeling Framework for experimental physics [13]. In the Modeling Framework, an experiment is divided into models of the measurement and physical system. In constructing

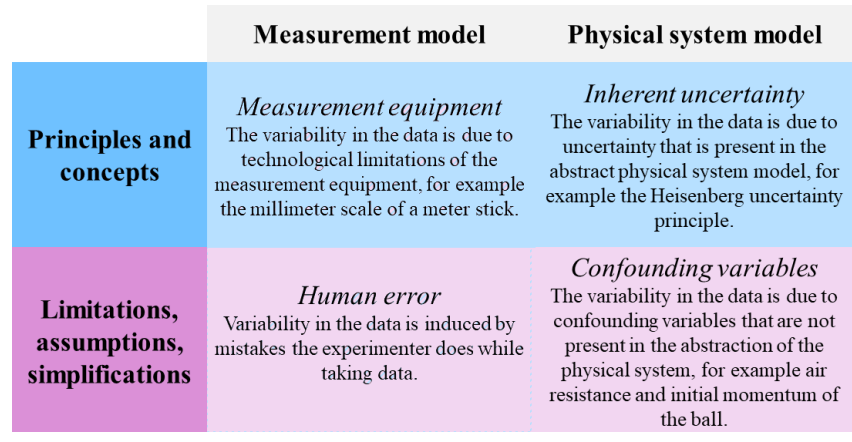

FIG. 2. Our coding scheme (italic) capturing the different sources of uncertainty that participants used to explain the variability in data and how these codes match the Modeling Framework [13] (bold).

these models, one must consider 1) the relevant principles and concepts and 2) the limitations and assumptions. Our codes shown in Fig. 2 broadly align with these categorizations.

\section{RESULTS}

We analyzed the data from the three interview questions and compare responses between the quantum and classical contexts. The following three sections each present the findings from one of these questions.

\section{A. Variability is attributed to experimental uncertainty in} classical and to inherent uncertainty in quantum mechanics

After showing participants each histogram in each context, we first asked them, "What can you say about the shape of the histogram?" and "Why does the distribution have the shape that it does?"

As evident from Fig. 3, most participants attributed the variability in the data of the ball-drop experiment to the influence of confounding variables: "Maybe they didn't put the ball at the same spot every time. Um, and maybe it's just stuff like that. It just didn't fall correctly. [...] there's just too many things to take into account. Like there's little bit of wind resistance." This was coded as human error (another common cause of uncertainty) in addition to confounding variables, because it is implied that the experimenters failed to put the ball in the same initial spot repeatedly. A third cause of uncertainty that was mentioned in response to this question was limitations to the measurement equipment: "There's a limitation of how accurately the meter stick can measure and there is a limitation of how well a student measures." We refer to these three sources of uncertainty as "experimental uncertainty'.

The most striking difference in answers to these questions in the single-slit experiment was that most participants attributed the variability in the data to uncertainty that is inherent to the theory, even though some participants admit- 


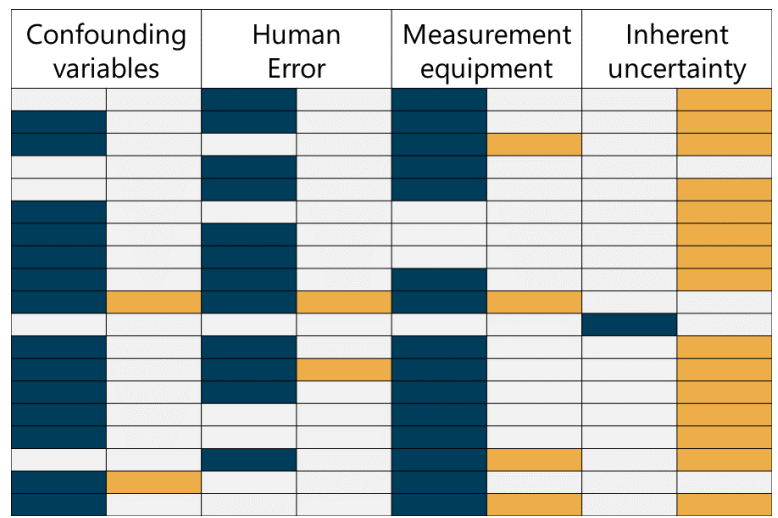

FIG. 3. Sources of uncertainty that are responsible for the shape of the histogram according to each participant. Each participant corresponds to one row. The first column under each source of uncertainty indicates whether that source was mentioned in classical mechanics, the second column whether it was mentioned in quantum mechanics. Blue/yellow means mentioned, grey means not mentioned.

ted that the data does not show the interference pattern they expected. Participants' answers varied greatly in the exact mechanism they used to explain the result and their sophistication. Some participants solely used wave effects to explain the results, others explicitly mentioned the wave-particle duality, and others relied on the Heisenberg uncertainty principle. One participant said that the histogram represents "the underlying wavefunction" and gave an explanation how the Heisenberg uncertainty principle increases the position uncertainty of the photons. Another participant said "as the photon goes through the single slit, the slit is going to, uh, diffract or it's going to change the angle of the photon. And so some of them, some of them can go straight through, but some of them will be angled a little up or a little down." While the participant seems to regard the photon as a particle moving straight or angled, it is guided by wave effects-diffraction.

What these answers have in common is that the uncertainty is created by the fundamental principles governing the physical system. Almost all (19/20) students said the variability in the data is coming from this inherent source of uncertainty. As evident from Figure 3, most participants did not mention uncertainty from any other sources in the context of the single slit experiment when explaining the shape of the histogram. This was rarely justified, although one participant mentioned the elimination of human interaction with the experimental setup eliminates experimental uncertainty: "I would say from my personal experiment experience of doing these I've been able to collect better data through the light experiment because there is less human interaction with that. Whereas with the ball drop, anything could happen in my personal opinion where you could drop the ball around and you can drop it not as high."

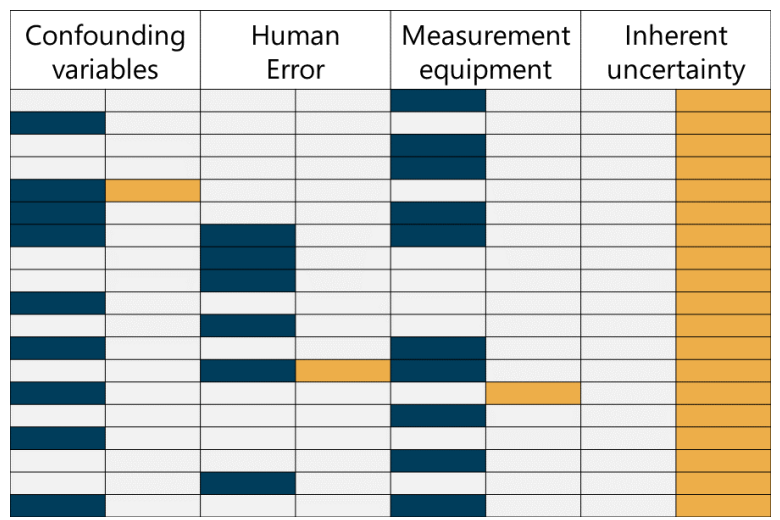

FIG. 4. Sources of uncertainty that participants mention when asked "What comes to mind when you think about measurement uncertainty in classical mechanics (quantum mechanics)?"

\section{B. An expert could reduce variability in classical mechanics} but not in quantum mechanics

We asked students: "If an expert scientist at a national laboratory would measure the distance of a ball after being released from the ramp (the positions of many photons on the screen after going through a single slit experiment) using high precision equipment, what would the histogram look like?"

In the ball-drop experiment, all but one participant argued that an expert scientist is able to reduce the width of the distribution. The sole participant stating that the width will stay the same argued "It's just science is messy". All others stated that the expert will be able to narrow down the distribution by using better measurement equipment or controlling confounding variables, to the point that it "might look like what we call a Delta function". These predictions of what the expert scientist would measure are consistent with students' responses to the first question. They further suggest that the variability in classical mechanics is coming from variables that are hard to control for students but manageable for an expert.

In the single-slit experiment, the answers to this question suggest that participants perceive the variability in the data as irreducible. Contrary to the ball-drop experiment, most participants said the histogram will not change its width, usually arguing that the histogram simply shows how waves or quantum mechanics works: "We expect to see the light photons hitting different places with some sort of probability spread because that's just the nature of how the light works." Some participants even said the distribution will get wider because more sensitive equipment might be able to pick up more photons at side maxima further out: "We would start to see these interference patterns happening out here." Only 4 students said that the distribution as measured by an expert might become narrower due to elimination of experimental uncertainty. 


\section{Some participants believe there is less experimental uncertainty in quantum mechanics}

In the middle and at the end of each interview, we asked participants, "What comes to mind when you think about measurement uncertainty in classical mechanics (quantum mechanics)?" The coding of all responses is presented in Fig. 4.

We are going to examine this question through one participant that we call Bob who made his reasoning very clear: "In classical mechanics, I mean everything that we do is on like a very, very-I mean compared to like anything small-very, very big in a sense. So our uncertainty in classical mechanics, it's like how high we dropped something, the angle at which we release a pendulum in a sense. And there's a lot of factors that could go wrong." Bob emphasizes how hard it is in his experience to repeat an experiment under the exact same conditions in classical mechanics, because there are so many factors that can go wrong. We coded this as attributing the uncertainty to confounding variables. Shortly after, he generalizes his statement to all his experiences with classical physics: "I feel like anytime that I do an experiment in classical physics, it's always going to be, there's always going to be a decent amount of error because there's so many things that are going on." Note how the participant associates the "error" in an experiment with things going "wrong", leaving open the possibility to fix these errors. As can be seen in Fig. 4, more than half of the participants similarly mentioned confounding variables that are not necessarily controlled for such as air resistance and imperfections in the ramp and the ball. Many others mentioned limitations of the measurement equipment such as the millimeter scale on a meter stick and human error when handling experimental equipment.

In quantum mechanics most students did not mention the effects of human error, measurement equipment or confounding variables, but only inherent uncertainty. Bob offers an explanation for this by contrasting his answer about quantum mechanics with his answer about classical mechanics: "[In] quantum mechanics there's a lot less variables involved because when you're just talking about like one atom, you don't have to worry about air resistance because it's pretty much nonexistent. You don't worry about gravity in a sense. [...] Classically you think, you know, oh, there's a distribution because we can't account for every single atom and what it's doing [...] but quantum mechanically you, you think, well, we're the smallest we can really get, we know every single factor about what's happening. So we should be able to predict absolutely what this atom is going to do. And it turns out that we really can't and it's a little unsettling and not very pleasing, but I think that's just something that I tend to accept a lot more in quantum mechanics then built into the system is uncertainty and it's not something that we can ever get better at." There is a strong contrast between experiments in classical mechanics where things that "go wrong" cause "errors" while in quantum mechanics the uncertainty "is built into the system" and is nothing "we can ever get better at".

\section{DISCUSSION}

We have shown that students differently interpret the variability of experimental data from specific experiments in classical and quantum mechanics. In a ball-drop experiment situated in the context of classical mechanics, students attribute the variability in the data to a variety of of factors including confounding variables, limitations to the measurement equipment, and other forms of human error. In the Modeling Framework of experimental physics, these factors are mostly associated with the limitations and simplifications to the physical and measurement system. On the other hand, in a single-photon-single-slit experiment most students attribute most of the variability to the inherent uncertainty of measurements in quantum mechanics. In the Modeling Framework, this corresponds to the principles and concepts of the physical system model. Hence, students conclude they (and even an expert) cannot reduce the variability in the data.

These results suggest that in quantum mechanics experiments students continue to evaluate variability from an abstract, theoretical perspective, rather than considering the experimental set up. Our interviews have shown that students do not recognize many sources of experimental uncertainty in quantum mechanics, even shortly after recognizing them in the context of classical mechanics.

Our results are in apparent contradiction to the results of Ayene et al (2011) [9] and Müller and Wiesner (2002) [10] who found that many students have not internalized the probabilistic nature of quantum mechanics. For example, both studies found that many students believe the Heisenberg uncertainty principle is a consequence of technical or random errors that happen during the measurement process, or due to physical disturbances caused by the measurement process. These students seem to believe that the uncertainty principle is merely a name for experimental sources of uncertainty in the context of quantum mechanics. This highlights a limitation of our study: We mostly probed which source of uncertainty come to students' minds, not necessarily how any source of uncertainty influences experimental data. For example, a student's answer that mentions the buzzword "Heisenberg uncertainty principle" would have been coded as inherent uncertainty, even if that student did not express an understanding of the uncertainty principle as encoding the probabilistic nature of quantum mechanics. However, our findings also add a layer of sophistication to previous findings: Regardless of how exactly students understand uncertainty in quantum mechanics data, they seem to believe that this uncertainty is (at least practically) irreducible as shown by the results of Section III B. This suggests students have given up the point-like notion of one "true" value in quantum mechanics measurements, a claim to be tested in future work.

\section{ACKNOWLEDGMENTS}

This work has been supported in part by the NSF under Grants No. DUE-1808945 and No. DUE-1809183. 
[1] N. Holmes and C. E. Wieman, Physical Review Physics Education Research 12, 020103 (2016).

[2] A. Buffler, S. Allie, and F. Lubben, International Journal of Science Education 23, 1137 (2001).

[3] B. Pollard, R. Hobbs, J. T. Stanley, D. R. Dounas-Frazer, and H. Lewandowski, 2017 PERC Proceedings (2018).

[4] S. Allie, A. Buffler, F. Lubben, and B. Campbell, in Research in Science Education - Past, Present, and Future (Kluwer Academic Publishers, Dordrecht, 2001), pp. 331-336, URL http://link.springer.com/10.1007/0-306-47639-8\{_\}47.

[5] S. McKagan, K. Perkins, and C. Wieman, Physical Review Special Topics-Physics Education Research 6, 020121 (2010).

[6] G. Zhu and C. Singh, Physical Review Special Topics-Physics Education Research 8, 010117 (2012).
[7] H. R. Sadaghiani and S. J. Pollock, Physical Review Special Topics-Physics Education Research 11, 010110 (2015).

[8] G. Passante, P. J. Emigh, and P. S. Shaffer, Physical Review Special Topics-Physics Education Research 11, 020111 (2015).

[9] M. Ayene, J. Kriek, and B. Damtie, Physical Review Special Topics-Physics Education Research 7, 020113 (2011).

[10] R. Müller and H. Wiesner, American Journal of physics 70, 200 (2002).

[11] C. Singh, American Journal of Physics 76, 400 (2008).

[12] T. C. Andrews and P. P. Lemons, CBEâLife Sciences Education 14, ar7 (2015).

[13] B. M. Zwick1, D. Hu, N. Finkelstein, and H. Lewandowski, Physical Review Special Topics-Physics Education Research 11, 020113 (2015). 